INPLASY

PROTOCOL

To cite: Wang et al. A comparison of qSOFA, NEWS and SIRS in predicting the accuracy of mortality in suspected sepsis patients: a meta-analysis. Inplasy protocol 202140029. doi:

10.37766/inplasy2021.4.0029

Received: 06 April 2021

Published: 06 April 2021

Corresponding author: Yu Zhao

zhaoyuu@hotmail.com

Author Affiliation:

Department of pharmacy, University Town Hospital Affiliated of Chongqing

Medical University, Chongqing 401331, China

Support: None.

Review Stage at time of this submission: Preliminary searches.

Conflicts of interest:

None declared.

\section{A comparison of qSOFA, NEWS and SIRS in predicting the accuracy of mortality in suspected sepsis patients: a meta-analysis}

Wang, C1; Hu, XL2; Xu, RF3; Zhang, R4; Zeng, YR5; Zhao Y6.

Review question / Objective: What is the best score in mortality prediction in adult patients with suspected sepsis, when comparing qSOFA, NEWS and SIRS?

Condition being studied: Sepsis is one of the significant causes of mortality. Sepsis-3 recommends the use of qSOFA to predict in-hospital mortality and prolonged intensive care unit stay in patients with suspected sepsis. However, qSOFA has always been controversial and it is not clear whether qSOFA, NEWS or SIRS is more accurate in predicting mortality.

Information sources: PubMed, EMbase, Web of Science, the Cochrane Library.

INPLASY registration number: This protocol was registered with the International Platform of Registered Systematic Review and Meta-Analysis Protocols (INPLASY) on 06 April 2021 and was last updated on 06 April 2021 (registration number INPLASY202140029).

\section{INTRODUCTION}

Review question / Objective: What is the best score in mortality prediction in adult patients with suspected sepsis, when comparing qSOFA, NEWS and SIRS?
Condition being studied: Sepsis is one of the significant causes of mortality. Sepsis-3 recommends the use of qSOFA to predict in-hospital mortality and prolonged intensive care unit stay in patients with suspected sepsis. However, qSOFA has always been controversial and it is not 
clear whether qSOFA, NEWS or SIRS is more accurate in predicting mortality.

\section{METHODS}

Participant or population: Inclusion: Adult patients with sepsis or suspected sepsis Exclusion: Obstetric patients.

Intervention: Suspected sepsis.

\section{Comparator: qSOFA, NEWS and SIRS.}

Study designs to be included: All types, excluding case reports.

Eligibility criteria: Inclusion criteria: (1)The study population was patients with suspected sepsis. (2)To evaluate or compare the accuracy of qSOFA, NEWS, and SIRS in predicting mortality. (3)Adequate information had to be provided to build a $2 \times 2$ contingency table (true positives [TP], false positives [FP], false negatives [FN], and true negatives [TN]). Exclusion criteria: Review articles, letters and conference abstracts.

Information sources: PubMed, EMbase, Web of Science, the Cochrane Library.

Main outcome(s): Mortality.

Quality assessment / Risk of bias analysis: Two investigators independently assessed the quality of the included studies according to the Quality Assessment for Diagnostic Accuracy Studies-2 (QUADAS-2). When there is a disagreement, a third researcher will join in to help solve the problem. The publication bias was analyzed using the Deek's funnel plot.

Strategy of data synthesis: Bivariate mixedeffects regression model was used to calculate the pooled estimates of sensitivity, specificity, and diagnostic odds ratio (DOR) with their standard errors and 95\% Cls. Hierarchical summary receiver operating characteristic (HSROC) curves were constructed to assess the overall diagnostic performance. We will compare the sensitivity and specificity of each score for mortality prediction. Heterogeneity was evaluated by $\mathrm{X} 2$ test. If $12 \leq 50 \%$ and $P \geq 0.1$, the heterogeneity among studies was acceptable. If $I 2>$ was $50 \%$ and $P<0.1$, the heterogeneity among the studies was large, and the source of heterogeneity was analyzed.

Subgroup analysis: None.

Sensitivity analysis: Sensitivity analysis was to exclude the included literatures one by one to assess the reliability and stability of the aggregated results.

Country(ies) involved: China.

Keywords: qSOFA, NEWS, SIRS, sepsis, mortality.

Contributions of each author:

Author 1 - Can Wang.

Author 2 - Xulian Hu.

Author 3 - Rufu Xu.

Author 4 - Yu Zhao.

Author 5 - Rong Zhang.

Author 6 - YueRong Zeng. 